\title{
Spontaneous Structural Rearrangements in Solanum phureja Juz. et Buk. 3. Gene-centromere mapping of ym (yellow margin) by half-tetrad analysis
}

\author{
M. Wagenvoort \\ DLO-Centre for Plant Breeding and Reproduction Research (CPRO-DLO), \\ P. O. Box 16,6700 AA Wageningen, The Netherlands
}

Accepted April 24, 1995

In diploid $(2 n=2 x=24)$ potatoes, several meiotic restitution mechanisms which can give rise to $2 n$ gametes have been found (Ramanna 1974, 1979, Mok and Peloquin 1975). Based on the genetic consequences, two distinct modes of $2 n$ gamete formation are distinguished: first division restitution (FDR) and second division restitution (SDR). Basically FDR $2 n$ gametes include non-sister chromatids, whereas SDR $2 n$ gametes comprise sister chromatids. Once the mode of $2 n$-gamete formation has been determined cytologically, half-tetrad analysis (HTA) can be used to map single genes with respect to the centromere (Mendiburu et al. 1974). HTA takes advantage of $2 n$-gamete formation in the diploid parent in $4 x-2 x, 2 x-4 x$ or $2 x-2 x$ matings.

In potato, a number of loci have been mapped by establishing the map distance to the centromere through interploidy crosses. A summary of relative map distances of morphological characters and isozymes reported in the literature has been presented by Wagenvoort and Zimnoch-Guzowska (1992). The morphological marker ym (yellow margin) was mapped 34.16 centimorgans $(\mathrm{cM})$ relative to the centromere via $4 x-2 x$ crosses, and $36.08 \mathrm{cM}$ via $2 x-4 x$ crosses (Jongedijk et al. 1991). The diploid parents used in these HTAs were synaptic and produced $2 n$ pollen by FDR or $2 n$ eggs by SDR.

The diploid clones of Solanum phureja Juz. et Buk. designated 1931 and 1936 are heterozygous for an interchange between chromosome 3 and chromosome 12 and for one or two paracentric inversions (Wagenvoort 1988, 1995). Clone 1936 produced seeds after selfing. This result suggested that the $S$ locus (gametophytic incompatibility) was involved in the interchange and had become inactive. Trisomics $(2 n=2 x+1=25)$ were found in the first inbred generation of 1936 and were identified as tertiary trisomics or primaries being homozygous for the interchange (Wagenvoort 1995).

To establish the genetic constitution of both the normal chromosomes 3 and 12 and the interchanged chromosomes $3^{12}$ and $12^{3}$ concerning the loci $S$ and $y m$, and with respect to the breakpoints $(\mathrm{T})$ of the interchange, it was necessary to ascertain the recombination rate of $y m$ in the interchange heterozygote. If this recombination value could be compared with the estimate made by Jongedijk et al. (1991), who used a different approach, conclusions regarding the degree of interference, if any between $y m$ and the centromere, could be drawn. In that case the relative map distance of $y m$ to the centromere estimated in the interchanged chromosome can be used in further genetic analysis of progenies of 1931 and 1936. Thus, $4 x \times 2 x$ testcrosses were performed for mapping the recessive gene $y m$ by HTA using the genotypes 1931 and 1936 .

In this paper the relative distance of $y m$ to the centromere in the interchange situation is estimated.

Telephone number: 0031837077060 Fax number: 0031837018094 E-mail: in\%"M.WAGENVOORT@CPRO.AGRO.NL" 
Materials and methods

\section{Plant material}

The diploid siblings $S$. phureja 75-1136-1931 and $S$. phureja 75-1136-1936, for the sake of brevity designated 1931 and 1936, were used in test-crosses and in HTA-mapping. Their pedigree has been described earlier in Wagenvoort (1988). The genotypes of these clones have been determined using the observed phenotype and the presence or absence of segregation in diploid progenies (Wagenvoort, unpublished results). Heterozygosity at the ym-locus in 1931 and 1936 was checked in the first inbred generation of 1936 and in an $F_{1}$ progeny of both clones. The diploid mutant for yellow margin, viz. Ym76-1-15 was selected from S. phureja (Wagenvoort 1982). From this clone a tetraploid $(2 n=4 x=48)$ was produced through tissue culture (Wagenvoort, unpublished results) using the method by Roest and Bokelmann (1976).

Crosses were made on plants grafted onto tomato root stocks and on plants grown on bricks in a temperature-conditioned glasshouse.

\section{Cytological methods and method of estimating gene-centromere map distance}

Ploidy level of $4 x-2 x$ progenies and of plants from tissue culture was determined by counting the number of plastids in the stomatal guard cells after staining with iodine-potassium iodine. Whenever in doubt the number of chromosomes was counted in root meristems according to the method described by Wagenvoort and Lange (1975). Study of various stages of meiosis in the diploids were carried out as described by Wagenvoort and Ramanna (1979) in order to ascertain the mode of $2 n$-pollen formation.

For estimating the map distance of $y m$ to the centromere in the interchange situation the $4 x-2 x$ cross was used since 1931 and 1936 produced $2 n$ gametes only at the male side.

Mendiburu and Peloquin (1979) assumed only single-exchanges to occur in potato. Under that assumption the proportion of single-exchange tetrads is a linear function of the map distance between the locus in question and the centromere. In case of FDR the genecentromere map distance $=2$ (frequency of nulliplex progeny) $\times 100 \mathrm{cM}$ (Mendiburn and Peloquin 1979). The binomial confidence interval was calculated using the expression

$$
\hat{p}-2 \sqrt{\frac{\hat{p} \hat{q}}{N}}<p<\hat{p}+2 \sqrt{\frac{\hat{p} \hat{q}}{N}}
$$

giving approximately $95 \%$ limits to $p$, where $\hat{p}$ and $\hat{q}$ are the frequencies of plants with and without the trait, respectively, and $N$ is the total number of plants (Clarke 1982).

Results

\section{Half-tetrad analysis with ym}

The diploid clones 1931 and 1936 were studied at MII and later stages of meiosis in order to study the mode of $2 n$-pollen formation. In Table 1 the frequencies of normal spindles, fused spindles and parallel spindles at MII are presented along with the distribution of the chromosomes at AII/TII and the dyad, triad and tetrad frequencies at the sporad stage. Fig. 1 shows fused spindles at MII. The relative contribution of fused spindles to the pool of big pollen grains in the clones 1931 and 1936 was 52.9 and $66.7 \%$ respectively, whereas the dyad frequency exceeded the frequency of fused spindles. Fig. 2 shows a part of a PMC with dyads only. Triads were found in 3-5\% of the PMCs analysed at the sporad stage. They will give rise to SDR or FDR $2 n$ pollen depending on whether one of the equational walls has failed to form or the reductional wall is partly formed respectively (Ramanna 1974). Variation in the frequency of dyads occurred within and between anthers of the same plant. Therefore, because 
Table 1. Frequencies of normal spindles (NS), parallel spindles (PS) and fused spindles (FS) at MII, chromosome distribution at AII/TII and the dyad, triad and tetrad frequency at the sporad stage in the diploid clones 1931 and 1936 from $S$. phureja Juz. et Buk.

\begin{tabular}{|c|c|c|c|c|c|c|c|c|c|c|c|c|}
\hline \multirow[b]{2}{*}{ Plant No. } & \multirow{2}{*}{$\begin{array}{l}\text { Number } \\
\text { of cells }\end{array}$} & \multicolumn{3}{|c|}{ MII (\%) } & \multirow{2}{*}{$\begin{array}{l}\text { Number } \\
\text { of cells }\end{array}$} & \multicolumn{3}{|c|}{ AII/TII (\%) } & \multirow{2}{*}{$\begin{array}{l}\text { Number } \\
\text { of cells }\end{array}$} & \multicolumn{3}{|c|}{ Sporad stage (\%) } \\
\hline & & NS & PS & FS & & $\begin{array}{l}12-12- \\
12-12\end{array}$ & $\begin{array}{l}12-12- \\
24\end{array}$ & $24-24$ & & tetrad & triad & dyad \\
\hline 1931 & 305 & 61 & 21 & 18 & 548 & 71 & 4 & 25 & 836 & 61 & 5 & 34 \\
\hline 1936 & 979 & 70 & 20 & 10 & 1400 & 70 & 9 & 21 & 1491 & 82 & 3 & 15 \\
\hline
\end{tabular}

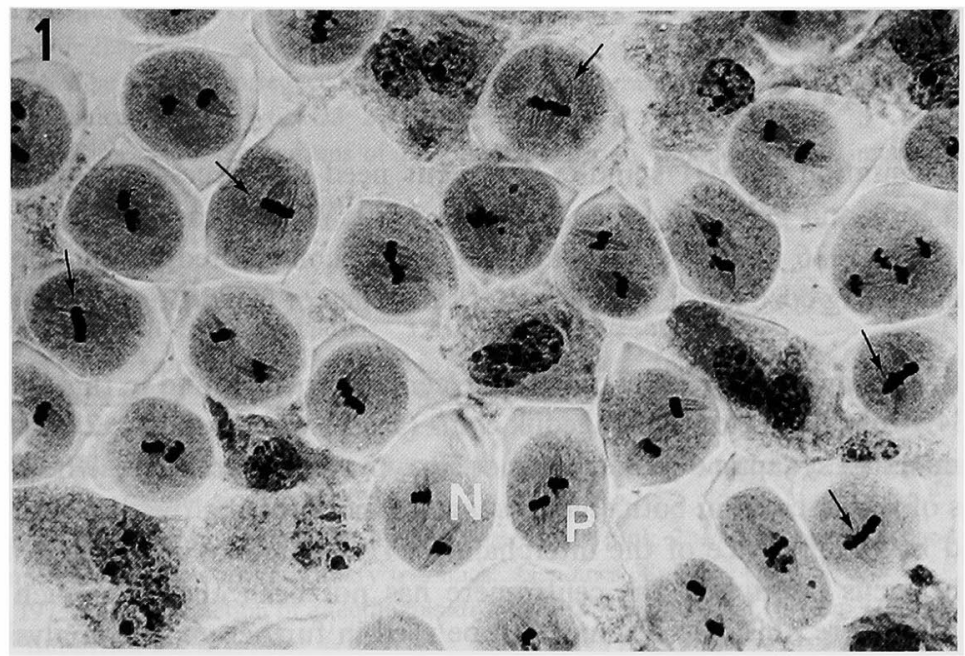

Fig. 1. PMCs showing spindles with normal orientation $(N)$, parallel orientation $(P)$ and fused spindles (arrows) at MII.

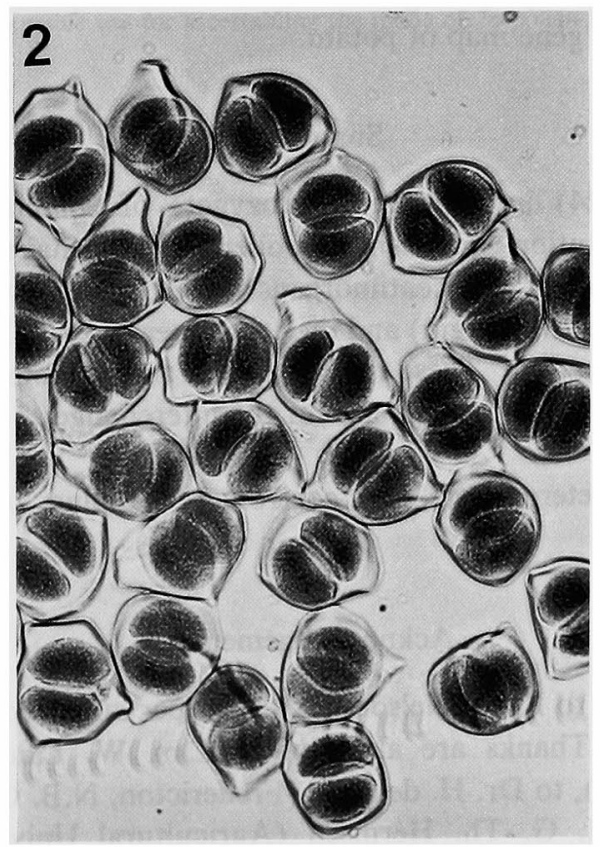

Fig. 2. Part of a PMC, showing dyads only. 
no other aberrations were observed, it was reasonable to conclude that fused and parallel spindles predominantly contributed to the pool of $2 n$ pollen of 1931 and 1936 , and consequently gave rise to FDR $2 n$ pollen.

The $4 x$ mutant for yellow margin was crossed to 1931 and 1936, the crosses being ymymymym $\times$ Ymym. The triploid offspring of these $4 x-2 x$ crosses segregated for $y m$ in 20 normal versus 26 mutant, which was consistent with the expected ratio $1: 1\left(\chi^{2}=0.78, P>\right.$ 0.30 ). In the tetraploid offspring the ratio 249 normal to 47 mutant was found. In the case of FDR $2 n$ pollen all mutants originate from crossing-over between $y m$ and the centromere. Using the formula of Mendiburu and Peloquin (1979) a map distance of $31.7 \mathrm{cM}$ was calculated with a $95 \%$ binomial confidence interval of $27.5-35.9 \mathrm{cM}$.

\section{Discussion}

Parallel orientation of MII spindles may, but need not necessarily result in 2 -pollen formation (cf. Mok and Peloquin 1975, Ramanna 1979, Wagenvoort 1986). Parallel spindles found in 1931 and 1936 in this study may have partly contributed to the pool of $2 n$-pollen, indicated by the strong correlation between the occurrence of fused/parallel spindles, and dyads (Table 2).

The relative distance of $y m(31.7 \mathrm{cM})$ to the centromere as found in this study was similar to that (viz. $34.16 \mathrm{cM}$ ) reported by Jongedijk et al. (1991) who in the $4 x-2 x$ testcross used the same $4 x$ mutant and a synaptic diploid clone not carrying an interchange. The similarity of the map distances of $y m$ assessed in both studies suggests that crossing over in 1931 and 1936 was not influenced by the presence of the interchange. This outcome also demonstrates that the position of the locus relative to the centromere has not been changed much owing to the interchange. Therefore, this map distance can be used in further genetic analysis of progenies from 1931 and 1936.

The relative map distance of $y m$ was successfully estimated by half-tetrad analysis and the mode of $2 n$-pollen formation by cytological analysis. This map distance can be used for the placement of the gene on a gene map of potato.

\section{Summary}

A diploid $(2 n=2 x=24)$ interchange heterozygote of Solanum phureja Juz. et Buk. produced first division restitution-equivalent $2 n$ pollen by abnormal meiosis II spindle orientation. A relative map distance of 31.7 centimorgans was estimated by a half-tetrad analysis for the distance between $y m$ (yellow margin) and the centromere. A similar distance was estimated for the non-interchange situation. These results suggest that crossing over in the interchange heterozygote is not influenced by the presence of the interchange.

Key words: interchange heterozygote, gene-centromere mapping, $2 n$ pollen, Solanum phureja Juz. et Buk.

\section{Acknowledgements}

The author is indebted to Karin Nelson, Greet Kuiper and Jacqueline de Haas-Buurman for technical assistance. Thanks are also due to Drs. W. Lange and J. Hoogendoorn (CPRO-DLO, Wageningen), to Dr. H. de Jong (Fredericton, N.B. Canada) and to Dr. M. S. Ramanna and Prof. Dr. J. G. Th. Hermsen (Agricultural University, Wageningen, The Netherlands) for critical comments on the manuscript. 


\section{References}

Clarke, G. M. 1982. Statistics and Experimental Design. The Camelot Press. Ltd., Southampton, United Kingdom, p. 79.

Jongedijk, E., Hutten, R. C. B., van der Wolk, J. M. A. S. A. and Schuurmans Stekhoven, S. E. J. 1991. Synaptic mutants in potato, Solanum tuberosum L. III. Effect of the $D s-1 / d s-1$ locus (desynapsis) on genetic recombination in male and female meiosis. Genome 34: 121-130.

Mendiburu, A. O. and Peloquin, S. J. 1979. Gene-centromere mapping by $4 x-2 x$ matings in potatoes. Theor. Appl. Genet. 54: 177-180.

Mendiburu, A. O., Peloquin, S. J. and Mok, D. W. S. 1974. Potato breeding with haploids and $2 n$ gametes. In: Haploids in Higher Plants: 249-258. Edited by K. Kasha. University of Guelph, Guelph, Ontario, Canada.

Mok, D. W. S. and Peloquin, S. J. 1975. Three mechanisms of $2 n$ pollen formation in diploid potatoes. Can. J. Genet. Cytol. 17: 217-225.

Ramanna, M. S. 1974. The origin of unreduced microspores due to aberrant cytokinesis in the meiocytes of potato and its genetic significance. Euphytica 23: 20-30.

- 1979. A re-examination of the mechanisms of $2 n$ gamete formation in potato and its implications for breeding. Euphytica 28: 537-561.

Roest, S. and Bokelmann, G. S. 1976. Vegetative propagation of Solanum tuberosum L. in vitro. Potato Res. 19: 173178.

Wagenvoort, M. 1982. Location of the recessive gene ym (yellow margin) on chromosome 12 of diploid Solanum tuberosum by means of trisomic analysis. Theor. Appl. Genet. 61: 239-243.

- 1986. The effect of temperature on dyad formation in Solanum phureja. In: Potato Research of Tomorrow: Drought Tolerance, Virus Resistance and Analytic Breeding Methods: 124-137. Pudoc, Wageningen, The Netherlands.

- 1988. Spontaneous structural rearrangements in Solanum tuberosum ssp. phureja: 1. Chromosome identification at pachytene stage. Euphytica 9: 159-167.

- 1995. Spontaneous structural rearrangements in $S$. phureja Juz. et Buk. 2. Meiotic behaviour and identification of interchange chromosomes using primary trisomics. Genomes 38: 140-147.

- and Lange, W. 1975. The production of aneudihaploids in Solanum tuberosum L. Group Tuberosum (the common potato). Euphytica 24: 731-741.

- and Ramanna, M. S. 1979. Identification of the trisomic series in diploid Solanum tuberosum L. Group Tuberosum. II. Trivalent configurations at pachytene stage. Euphytica 28: 633-642.

- and Zimnoch-Guzowska, E. 1992. Gene-centromere mapping in potato by half-tetrad analysis: map distances of $\boldsymbol{H}_{1}$, $R x$ and $R y$ and their possible use for ascertaining the mode of $2 n$-pollen formation. Genome 35: 1-7. 